\title{
O efeito da restrição calórica na longevidade
}

\author{
Effect of caloric restriction on longevity
}

Patrícia de Souza Genaro', Karin Sedó Sarkis' ${ }^{1}$ Ligia Araújo Martini'

\section{RESUMO}

A restrição calórica $(R C)$ é uma das formas de intervenção nutricional mais amplamente discutida para se estender o tempo de vida em uma variedade de espécies, inclusive seres humanos. A RC parece reduzir a incidência de doenças relacionadas à idade. 0 mecanismo clássico que poderia explicar o efeito do consumo calórico no envelhecimento está relacionado à redução da gordura corporal e à sinalização da insulina, somada às espécies reativas de oxigênio produzidas durante a respiração que causam danos oxidativos ao DNA e ao RNA das células, promovendo o processo de envelhecimento e o aumento do risco de doenças. No entanto, o efeito da RC na longevidade em humanos ainda não está bem estabelecido e mais estudos são necessários para que os mecanismos celulares e moleculares responsáveis pelos efeitos terapêuticos da restrição calórica sejam elucidados. Além disso, é necessário diferenciar os efeitos benéficos da restrição calórica daqueles relacionados a hábitos alimentares saudáveis. Arq Bras Endocrinol Metab. 2009;53(5):667-72.

Descritores

Restrição calórica; envelhecimento; estresse oxidativo; doenças crônicas
${ }^{1}$ Faculdade de Saúde Pública, Universidade de São Paulo (FSP/USP), São Paulo,SP, Brasil

\begin{abstract}
Calorie restriction $(\mathrm{CR})$ is the most evaluated nutritional intervention to increase lifespan in a variety of animal species, including human beings. CR has also been shown to delay the onset or reduce the incidence of many age-related diseases. The mechanism that could explain the effect of calorie intake on aging is related to the reduction of body fat and insulin signaling as well as reactive oxygen species produced during breathing. These phenomena cause oxidative damage to DNA and RNA promoting the process of aging and increasing the risk of illnesses. However, the effect of $\mathrm{CR}$ on longevity in human beings is not fully established and further studies are necessary in order to identify the molecular and cellular mechanisms for the therapeutic effect of RC. Moreover, it is necessary to set up the differences between the beneficial effects of caloric restriction from those related to dietary healthy habits. Arq Bras Endocrinol Metab. 2009;53(5):667-72.

Keywords

Caloric restriction; aging; oxidative stress; chronic disease
\end{abstract}

Correspondência para: Ligia Araújo Martini Departamento de Nutrição, Faculdade de Saúde Pública da Universidade de São Paulo Av. Doutor Arnaldo, 715, 2ㅇadar 01246-904 - São Paulo, SP, Brasil Imartini@usp.br

Recebido em 12/Mai/2009 Aceito em 18/Mai/2009

\section{INTRODUÇÃO}

$\mathrm{O}$ processo de envelhecimento é complexo e associase a uma perda gradual das funções fisiológicas regulada por fatores genéticos e ambientais. A restrição calórica (RC), definida como uma redução da ingestão calórica abaixo do ad libitum, sem desnutrição, é uma das formas de intervenção nutricional mais amplamente discutidas para se estender o tempo de vida em uma variedade de espécies, inclusive mamíferos (1-3).

Estudos em modelos animais associam a RC a uma menor incidência de doenças relacionadas à idade, tais como doenças cardiovasculares, câncer e diabetes (1-3), no entanto, os efeitos da RC em seres humanos ainda não estão bem estabelecidos $(4,5)$. Sendo assim, esta revisão visa a elucidar as principais evidências dos efeitos da RC sobre a longevidade do ponto de vista endocrinometabólico.

\section{HIPÓTESES DO MECANISMO DE ATUAÇÃO DA RESTRIÇÃO CALÓRICA NA LONGEVIDADE}

O mecanismo biológico responsável pelo efeito da restrição calórica na longevidade ainda é desconhecido, no entanto, algumas hipóteses têm sido propostas, tais 
como: hipótese da redução da gordura corporal e sinalização da insulina, e hipótese da redução da produção de espécie reativa de oxigênio e atenuação dos danos oxidativos (6).

\section{Hipótese da redução da gordura corporal e sinalização da insulina}

A alteração fisiológica, importante durante a restrição calórica, é iniciada com a redução da concentração de glicose no sangue ocasionada pela baixa ingestão de energia proveniente da dieta. Isto levará a uma diminuição da produção de insulina pelas células $ß$ do pâncreas e, consequentemente, a uma diminuição do depósito de tecido adiposo, principalmente tecido adiposo branco (7).

Pesquisas recentes têm demonstrado que o depósito de gordura (tecido adiposo branco) tem outras funções além de estocar gordura $(8,9)$. Tecido adiposo branco é um órgão endócrino, que produz hormônios ativos em todo o organismo como: fator de necrose tumoral- $\alpha$ (TNF- $\alpha$ ), resistina, adiponectina e leptina (10). A alteração do depósito de gordura, principalmente o tecido adiposo branco, poderia modificar a secreção desses hormônios, como liberar maior concentração de adiponectina e menor concentração de TNF- $\alpha$, melhorando a sensibilidade à insulina em diversos tecidos, como o muscular $\mathrm{e}$ o hepático. Essas mudanças endocrinometabólicas poderiam promover maior expectativa de vida $(11,12)$.

\section{Hipótese da redução da produção de espécie reativa de oxigênio e atenuação dos danos oxidativos}

As espécies reativas de oxigênio são formadas pela respiração celular. De todo o oxigênio utilizado pelas células na respiração, cerca de $2 \%$ dos átomos ficarão apenas parcialmente reduzidos por aceitar um só elétron. As principais espécies reativas de oxigênio resultantes são o ânion superóxido $\left(\mathrm{O}_{2}{ }^{-}\right)$, o peróxido de hidrogênio $\left(\mathrm{H}_{2} \mathrm{O}_{2}\right)$ e o radical hidroxil $\left(\mathrm{OH}^{-}\right)$que muito reativas e que, por isso, oxidam parcialmente com outras moléculas que se encontrem próximas, tais como lipídeos, proteínas ou ácidos nucleicos. Além disso, as espécies reativas de oxigênio ativam um fator transcricional pró-inflamatório denominado $\mathrm{NF}-\kappa \mathrm{B}$, responsável pela transcrição de proteínas pró-inflamatórias como TNF- $\alpha$ e inteterleucinas 1, 2 e 6. (13-15).

Os danos oxidativos, como a ativação de genes próinflamatórios, causados por essas espécies reativas de oxigênio estão fortemente relacionados ao envelhecimento e à patogênese de diversas doenças crônicas não transmissíveis, como aterosclerose, diabetes, artrite reumatoide, desordens neurodegenerativas e câncer (16).

$\mathrm{O}$ mecanismo pelo qual a restrição calórica diminui a formação das espécies reativas de oxigênio ainda não está estabelecido; no entanto, a RC parece promover melhora nos danos oxidativos, como a supressão da expressão e a ativação do NF- $\kappa$ B. Além disso, melhora o sistema de reparação do DNA celular (15).

\section{TEORIAS MOLECULARES DO MECANISMO DE ATUAÇÃO DA RESTRIÇÃO CALÓRICA NA LONGEVIDADE}

Muitos dos efeitos da restrição calórica parecem ser mediados pela regulação dos genes envolvidos no reparo celular, na resistência ao estresse e na proteção contra danos oxidativos, assim como genes responsáveis pela redução na mediação da inflamação e na prevenção de algumas alterações da expressão gênica que ocorrem com a idade $(17,18)$.

Algumas teorias surgiram e vários caminhos de sinalização celular têm sido relacionados à longevidade. Uma das primeiras vias de sinalização proposta foi a diminuição das concentrações de glicose determinada pela adenosina monofosfato cíclico (AMP cíclico), dependente da via da proteína quinase A (PKA), a qual sinaliza a disponibilidade da glicose para células (19).

Outra via de sinalização foi encontrada em leveduras, nas quais se descobriu que o efeito determinante da longevidade era mediado pela indução de um gene chamado silent information regulator 2 (regulador de informação silenciosa, ou apenas Sir2) que codifica uma enzima, a histona desacetilase dependente de nicotinamida adenina dinucleotídeo (NAD+) (20). Estudos de proteômica comparada mostraram que a Sir2 pertence a uma classe de proteínas chamadas sirtuínas. Em mamíferos, há sete genes de sirtuínas, e a sirtuína 1 (SIRTl) é a mais parecida com $\operatorname{Sir} 2(21)$.

Sabe-se que, em mamíferos, a restrição calórica aumenta as concentrações de SIRTl e sua expressão, enquanto a atividade biológica parece mediar a atividade de importantes reguladores de transcrição do metabolismo como forkhead transcription factor (FOXOl), peroxisome prolifarator-activated receptor $\alpha$ (PPAR $\alpha)$, peroxisome prolifarator-activated receptor $\gamma$ (PPAR $\gamma$ ), PPAR $\gamma$ coactivator- $1 \alpha$ (PGC-l $\alpha$ ) (22). No figado, a SIRTI desacetecila e ativa PGC-1 $\alpha$, que interage com FOXO1, no qual induz a ativação da gliconeogênese e diminui a glicólise (23). No tecido muscular, a SIRTI 
ativa PGC- $1 \alpha$, induzindo a biogênese mitocondrial e aumentando a oxidação de ácidos graxos (23). No tecido adiposo branco, suprime a atividade transcricional do PPAR $\gamma$, levando ao aumento da mobilização de gordura e à diminuição da adipogênese (24). As SIRT3 e SIRT4 parecem desempenhar papéis importantes no funcionamento da mitocôndria. Na presença de restrição calórica, ocorre a ativação da nicotinamide phosphoribosyltransferase (NAMPT) que catalisa a síntese de nicotinamida adenina dinucleotídeo (NAD). Concentrações elevadas de NAMPT levam ao aumento mitocondrial de $\mathrm{NAD}^{+}$que promove a proteção ao estresse genotóxico e morte celular (24). O papel preciso das sirtuínas na regulação da longevidade ainda não está totalmente elucidado, mas os estudos realizados até agora mostraram que essas proteínas apresentam papel fundamental na determinação da longevidade.

\section{RESTRIÇÃO CALÓRICA EM MODELOS ANIMAIS}

Em 1935, McCay e cols. publicaram pela primeira vez o efeito da restrição calórica (RC) em ratos. O estudo observou que, quando a restrição calórica era executada após a puberdade, os ratos apresentavam vida prolongada e atenuação das severidades de doenças crônicas (25).

Sabe-se que, em roedores, a redução calórica de $30 \%$ a $60 \%$ menos que o ad libitum imediatamente após a puberdade (seis meses) causou um aumento proporcional de $30 \%$ a $60 \%$ na expectativa de vida máxima, enquanto uma redução de $44 \%$ na ingestão calórica na idade adulta (12 meses) estendeu a expectativa de vida máxima em somente $10 \%$ a $20 \%$ (26).

Estudos conduzidos em camundongos atestaram que a restrição calórica aumenta a longevidade impedindo ou retardando a ocorrência das doenças crônicas como: arteriosclerose, cardiomiopatia, diabetes, doenças autoimunes, câncer, Alzheimer e Parkinson (27-31).

Camundongos transgênicos com elevada concentração de hormônio do crescimento mostraram que a restrição calórica parece promover alterações nas adipocinas, pois foram observados aumento da adiponectina e diminuição nos níveis séricos de interleucina-6 (IL-6), triacilgliceróis, colesterol e melhor sensibilidade à insulina (27). Outro estudo realizado por Guo e cols., em 2002, observou que camundongos homozigotos para a apolipoproteína E (ApoE (-/-)) que se alimentavam com uma dieta restritiva em calorias $(60 \%$ menos que o ad libitum) tinham diminuição significativa nas lesões ateroscleróticas quando comparados a camundongos que não foram submetidos à restrição calórica. Além disso, camundongos (ApoE (-/-)) com restrição calórica apresentavam diminuição significativa do estresse oxidativo na parede da aorta, contribuindo para um efeito antiaterogênico (28).

Estudos avaliando o efeito da restrição calórica no sistema imune parecem ser dependentes da ativação de linfócito $\mathrm{T}$ e macrófagos, pois ambos são responsáveis pelo tipo e extensão da resposta imune. Em modelos animais, a restrição calórica parece retardar o início das doenças autoimunes dependentes de linfócito-T e macrófagos como artrite reumatoide e lúpus eritomatoso sistêmico. Estudo em modelo animal para lúpus eritomatoso sistêmico e com doença renal como desfecho observou que a restrição calórica de $40 \%$, quando iniciada com seis semanas de vida, retardava o aparecimento da doença renal autoimune em $30 \%$ (29).

A restrição calórica parece diminuir a neurodegeneração no cérebro causada pela doença de Alzheimer e Parkinson (30,31). Estudo realizado em primatas induzidos à doença de Parkinson demonstrou menor déficit motor naqueles com restrição calórica de 30\% quando comparados a primatas que ingeriram uma dieta normal. $\mathrm{Na}$ análise neuroquímica, observou-se também que as concentrações de dopamina e de dois de seus metabólitos estavam mais elevadas no grupo com restrição calórica (30).

Patel e cols. examinaram o efeito da restrição calórica com duração de 6 a 14 semanas em camundongos transgênicos para a doença de Alzheimer. Houve diminuição substancial de $50 \%$ na imunorreatividade da beta-amioide, peptídeo que se acumula na massa cerebral nesta doença (31).

\section{RESTRIÇÃO CALÓRICA EM HUMANOS}

Ainda não está estabelecido se a restrição calórica teria o mesmo efeito benéfico sobre a longevidade de humanos que tem sobre os animais. Sabe-se que a restrição calórica dispara um mecanismo de proteção da vida em animais de vida curta, tais como roedores, que permite que sobrevivam a períodos de escassez alimentar. Contudo, a existência desse mesmo mecanismo em humanos não é conhecida. Entretanto, a escassez de comida durante a Segunda Guerra Mundial foi associada a uma diminuição de mortalidade por doença coronariana em países europeus (32).

Um estudo foi feito na Biosfera 2, local projetado para ser autossuficiente e ecologicamente controlado, 
em que oito participantes ficaram enclausurados durante dois anos. Durante 18 meses, esses participantes tiveram uma dieta com restrição da ingestão calórica de $22 \%$ (2.500 kcal/dia para $1.925 \mathrm{kcal} /$ dia). Observou-se redução do índice de massa corporal, além de redução de fatores de risco metabólicos para doenças coronarianas, tais como perfil lipídico e pressão sanguínea (33).

Resultados de estudos (34-36) realizados nos membros da Caloric Restriction Society, isto é, pessoas que fazem restrição calórica por livre arbítrio, foram publicados recentemente. O grupo de restrição calórica tinha um consumo de $1.800 \mathrm{kcal} / \mathrm{dia}$ (30\% menos caloria quando comparado à dieta típica americana, pareado para sexo e idade). Essa limitação durou aproximadamente seis anos e meio. Além da restrição calórica, era realizada uma dieta baseada na alta ingestão de frutas, vegetais, leite e derivados, proteína derivada da soja e baixo consumo de alimentos refinados. Os dados foram comparados com os dados de um grupo que seguia uma alimentação típica americana. Foi observado que o grupo com restrição calórica tinha menor porcentagem de gordura corporal, pressão sanguínea sistólica e diastólica menor, assim como melhor perfil lipídico, aumento da sensibilidade à insulina, baixos níveis de marcadores inflamatórios e baixa concentração de triiodotironina.

Um programa de pesquisa denominado CALERIE (Comprehensive Assessment of Long-Term Effects of Reducing Calorie Intake), ainda em andamento, que envolve três centros de estudos: Tufts University, Pennington Biomedical Research Center e Washington University, está sendo realizado a fim de estudar melhor o efeito da restrição calórica em humanos (37-41). A fase 1 do estudo consiste em três estudos pilotos para determinar a viabilidade de se investigar o efeito da restrição calórica em pessoas da comunidade, vivendo livremente. $\mathrm{O}$ estudo na Universidade de Tufts teve 12 meses de duração com $25 \%$ de restrição calórica da dieta, com indivíduos (homens e mulheres) entre 24 e 42 anos de idade, saudáveis e com sobrepeso (IMC 25 a $29,9 \mathrm{~kg} / \mathrm{m}^{2}$ ). O estudo em Pennington também teve 12 meses de duração com $25 \%$ de restrição calórica em homens e mulheres saudáveis entre 25 e 50 anos de idade. O estudo da Universidade de Washington envolveu homens e mulheres saudáveis e com sobrepeso, entre 50 e 60 anos de idade e com duração de 12 meses com $20 \%$ de restrição calórica. Os resultados da fase 1 em Pennington, após seis meses de duração, apenas apresentaram diminuição de $10 \%$ do peso corporal, diminuição significativa da temperatura corporal, na triiodotiroxina (T3), gordura corporal, tecido adiposo visceral e tecido adiposo subcutâneo, tamanho da célula adiposa, gordura intra-hepática, insulina de jejum e melhora significativa da sensibilidade à insulina (37-39). Os resultados da fase 1 em Washington com 12 meses de duração foram: redução de $10,7 \%$ no peso corporal e diminuição do IMC de 27,2 para $24,4 \mathrm{~kg} / \mathrm{m}^{2}$. Houve também uma redução significativa da gordura corporal, gordura visceral, gordura subcutânea abdominal, concentração de leptina, insulina e glicemia de jejum. A sensibilidade à insulina também aumentou significativamente em resposta à restrição calórica $(40,41)$.

A fase 2 do projeto CALERIE envolvendo os mesmos centros de pesquisas já está em andamento. Essa fase terá duração de 24 meses e uma maior inclusão de indivíduos eutróficos e saudáveis, com idade entre 25 e 40 anos, com $25 \%$ de restrição calórica. Os resultados dessas pesquisas serão finalizados no ano de 2011.

Embora a restrição calórica pareça ser benéfica e os resultados fornecidos pelos estudos demonstrem vantagens para o sistema cardiovascular e metabolismo da glicose e lipídeos, um estudo avaliando a massa óssea observou diminuição significativa da densidade mineral óssea total do quadril $(-2,2 \%)$ e na densidade mineral óssea da coluna lombar $(-2,2 \%)$ em comparação a indivíduos saudáveis (42).

A restrição calórica está associada, ainda, à diminuição no metabolismo energético e à redução da taxa do metabolismo basal; no entanto, esse efeito é controverso, pois poderia estar relacionado à perda de peso e adaptação metabólica dos tecidos muscular e adiposo (43). Além disso, não é possível determinar um ponto de corte seguro para restrição calórica, uma vez que há diferentes fatores que a influenciam como, por exemplo: composição corporal, diferença do gasto energético total segundo estágio de vida e gênero e a duração da restrição calórica (44).

Adicionalmente, para se avaliar o real efeito da restrição calórica na longevidade, seria necessário caracterizar e identificar novos marcadores do envelhecimento que estejam relacionados à adaptação do sistema neuroendócrino, prevenção da inflamação e proteção contra os prejuízos do estresse oxidativo que poderiam ajudar a predizer morbidade e mortalidade na população em geral (45).

Por outro lado, os efeitos benéficos observados nos estudos em humanos podem também estar relacionados a hábitos alimentares e de vida mais saudáveis. Portanto, seria necessário comparar, idealmente por meio de ensaios 
clínicos randomizados, o efeito da redução na ingestão alimentar e a alteração na qualidade da dieta, uma vez que ambos parecem ser concomitantes. Mesmo que a restrição calórica pareça aumentar a expectativa de vida e maximizar a longevidade humana, a adesão à restrição calórica por período de tempo prolongado seria incerta (44).

\section{CONCLUSÃO}

$\mathrm{O}$ efeito da restrição calórica na longevidade em humanos ainda não está bem estabelecido. As informações disponíveis sugerem que a redução de $20 \%$ a $30 \%$ de calorias na dieta parece diminuir o risco de desenvolvimento de doenças crônicas não transmissíveis; no entanto, mais estudos são necessários para que os mecanismos celulares e moleculares responsáveis pelos efeitos terapêuticos da restrição calórica sejam identificados. Além disso, é necessário diferenciar os efeitos benéficos da restrição calórica daqueles relacionados a hábitos alimentares e de vida saudáveis.

Declaração: os autores declaram não haver conflitos de interesse científico neste estudo.

\section{REFERÊNCIAS}

1. Roth GS, Ingram DK, Lane MA. Caloric restriction in primates and relevance to humans. Ann NY Acad Sci. 2001;928:305-15.

2. Weindruch R, Sohal RS. Caloric intake and aging. N Engl J Med. 1997;337(14):986-94.

3. Bordone L, Guarente L. Calorie restriction, SIRT1 and metabolism: understanding longevity. Nat Rev Mol Cell Biol. 2005;6(4):298-305.

4. Heilbronn LK, Ravussin E. Calorie restriction and aging: review of the literature and implications for studies in humans. Am J Clin Nutr. 2003;78(3):361-9.

5. Ingram DK, Roth GS, Lane MA, Ottinger MA, Zou S, de Cabo R, et al. The potential for dietary restriction to increase longevity in humans: extrapolation from monkey studies. Biogerontology. 2006;7(3):143-8.

6. Masoro EJ. Overview of caloric restriction and ageing. Mech Ageing Dev. 2005;126(9):913-22.

7. Koubova J, Guarente L. How does calorie restriction work? Gen Dev. 2003;17(3):313-21.

8. Barzilai N, Gupta G. Revisiting the role of fat mass in the life extension induced by caloric restriction. J Gerontol A Biol Sci Med Sci. 1999;54(3):B89-96.

9. Kissebah $\mathrm{AH}$. Insulin resistance in visceral obesity. Int $\mathrm{J}$ Obes. 1991;15(Suppl 2):109-15.

10. Bjorntorp P. Metabolic implications of body fat distribution. Diabetes Care. 1991;14(12):1132-43.

11. Wu X, Motoshima H, Mahadev K, Stalker TJ, Scalia R, Goldstein BJ. Involvement of AMP-activated protein kinase in glucose uptake stimulated by the globular domain of adiponectin in primary rat adipocytes. Diabetes. 2003;52(6):1355-63.

12. Combs TP, Berg AH, Rajala MW, Klebanov $S$, lyengar $P$, JimenezChillaron JC, et al. Sexual differentiation, pregnancy, calorie restriction, and aging affect the adipocyte-specific secretory protein adiponectin. Diabetes. 2003;52(2):268-76.
13. Sohal RS, Weindruch R. Oxidative stress, caloric restriction, and aging. Science. 1996;273(5271):59-63.

14. Dröge W. Free radicals in the physiological control of cell function. Physiol Rev. 2002;82(1):47-95.

15. Yu BP. Why calorie restriction would work for human longevity. Biogerontology. 2006;7(3):179-82.

16. Raha S, Robinson BH. Mitochondria, oxygen free radicals, disease and ageing. Trends Biochem Sci. 2000;25(10):502-8.

17. Weraarchakul N, Strong R, Wood WG, Richardson A. Effect of aging and dietary restriction on DNA repair. Exp Cell Res. 1989;181(1):197-204.

18. Alexeyev MF, Ledoux SP, Wilson GL. Mitochondrial DNA and aging. Clin Sci (Lond). 2004;107(4):355-64.

19. Thevelein JM, Winde JH. Novel sensing mechanisms and targets for the cAMP-protein kinase A pathway in the yeast Saccharomyces cerevisiae. Mol Microbiol.1999;33(5):904-18.

20. Landry J, Sutton A, Tafrov ST, Heller RC, Stebbins J, Pillus L, et al.The silencing protein SIR2 and its homologs are NAD-dependent protein deacetylases. Proc Natl Acad. Sci USA. 2000;97(11):5807-11.

21. Kaeberlein M, Kirkland KT, Fields S, Kennedy BK. Sir2-independent life span extension by calorie restriction in yeast. PLoS Biol. 2004;2(9):1381-7.

22. Bordone L, Guarente L. Calorie restriction, SIRT1 and metabolism: understanding longevity. Nat Rev Mol Cell Biol. 2005;6(4):298-305.

23. Haigis MC, Guarente LP. Mammalian sirtuins - emerging roles in physiology, aging, and calorie restriction. Genes Dev. 2006;20(21):2913-21.

24. Kurtev M, Chung N, Topark-Ngarm A, Senawong T, Machado De Oliveira $\mathrm{R}$, et al. Sirt1 promotes fat mobilization in white adipocytes by repressing PPAR. Nature. 2004;429(6993):771-6.

25. McCay CM, Crowel MF, Maynard LA. The effect of retarded growth upon the length of the life span and upon the ultimate body size. J Nutr. 1935; 10:63-79.

26. Weindruch $R$, Walford RL. Dietary restriction in mice beginning at 1 year of age: effect on life-span and spontaneous cancer incidence. Science. 1982;15(4538):1415-8.

27. Wang Z, Masternak MM, Al-Regaiey KA, Bartke A. Adipocytokines and the regulation of lipid metabolism in growth hormone transgenic and calorie-restricted mice. Endocrinology. 2007;148(6):2845-53.

28. Guo Z, Mitchell-Raymundo F, Yang H, Ikeno Y, Nelson J, Diaz V, et al. Dietary restriction reduces atherosclerosis and oxidative stress in the aorta of apolipoprotein E-deficient mice. Mech Ageing Dev. 2002;123(8):1121-31.

29. Jolly CA, Muthukumar A, Avula CP, Troyer D, Fernandes G. Life span is prolonged in food-restricted autoimmune-prone (NZB $x$ $\mathrm{NZW}$ ) $\mathrm{F}(1)$ mice fed a diet enriched with ( $\mathrm{n}-3)$ fatty acids. J Nutr. 2001;131(10):2753-60.

30. Maswood N, Young J, Tilmont E, Zhang Z, Gash DM, Gerhardt $\mathrm{GA}$, et al. Caloric restriction increases neurotrophic factor levels and attenuates neurochemical and behavioral deficits in a primate model of Parkinson's disease. Proc Natl Acad Sci USA. 2004;101(52):18171-6.

31. Patel NV, Gordon MN, Connor KE, Good RA, Engelman RW, Mason $\mathrm{J}$, et al. Caloric restriction attenuates Ab-deposition in Alzheimer transgenic models. Neurobiol Aging. 2005;26(7):995-1000.

32. Hautvast JG. The future of nutrition in Europe. Eur J Clin Nutr. 1993;47(Suppl 1):S96-100.

33. Walford RL, Mock D, Verdery R, MacCallum T. Calorie restriction in biosphere 2: alterations in physiologic, hematologic, hormonal, and biochemical parameters in humans restricted for a 2-year period. J Gerontol A Biol Sci Med Sci. 2002;57(2):B211-24.

34. Fontana $\mathrm{L}$, MeyerTE, Klein S, Holloszy JO. Long-term calorie restriction is highly effective in reducing the risk for atherosclerosis in humans. Proc Natl Acad Sci USA. 2004;101(17):6659-63. 
35. MeyerTE, Kovacs SJ, Ehsani AA, Klein S, Holloszy JO, Fontana L. Long-term caloric restriction ameliorates the decline in diastolic function in humans. J Am Coll Cardiol. 2006;47(2):398-402.

36. Fontana L, Klein S, Holloszy JO, Premachandra BN. Effect of long-term calorie restriction with adequate protein and micronutrients on thyroid hormones. J Clin Endocrinol Metab. 2006;91(8):3232-5.

37. Redman LM, Heilbronn LK, Martin CK, Alfonso A, Smith SR, Ravussin $\mathrm{E}$, for the Pennington CALERIE Team. Effect of calorie restriction with or without exercise on body composition and fat distribution. J Clin Endocrinol Metab. 2007;92(3):865-72.

38. Heilbronn LK, Jonge L, Frisard MI, DeLany JP, Larson-Meyer E, Rood J, et al; for the Pennington CALERIETeam. Effect of 6-month calorie restriction on biomarkers of longevity, metabolic adaptation, and oxidative stress in overweight individuals: a randomized controlled trial. JAMA. 2006;295(13):1539-48.

39. Larson-Meyer DE, Heilbronn LK, Redman LM, Newcomer BR, Frisard $\mathrm{MI}$, Anton S, et al. Effect of calorie restriction with or without exercise on insulin sensitivity, b-cell function, fat cell size, and ectopic lipid in overweight subjects. Diabetes Care. 2006;29(6):1337-44.
40. Racette SB, Weiss EP, Villareal DT, Arif H, Steger-May K, Schechtman $K B$, et al. One year of caloric restriction in humans: feasibility and effects on body composition and abdominal adipose tissue. J Gerontol Med Sci. 2006;61(9):943-50.

41. Weiss EP, Racette SB, Villareal DT, Fontana L, Steger-May K, Schechtman $\mathrm{KB}$, et al. Improvements in glucose tolerance and insulin action induced by increasing energy expenditure or decreasing energy intake: a randomized controlled trial. Am J Clin Nutr. 2006;84(5):1033-42.

42. Villareal DT, Fontana L, Weiss EP, Racette SB, Steger-May K, Schechtman KB, et al. Bone mineral density response to caloric restriction-induced weight loss or exercise induced weight loss. Arch Intern Med. 2006;166(22):2502-10.

43. Redman LM, Heilbronn LK, Martin CK, Jonge L, Williamson DA, Delany JP, et al. Behavioral compensations in response to caloric restriction: implications for the maintenance of weight Loss. PLoS ONE. 2009;4(2):1-9.

44. Fontana L, Klein S. Aging, adiposity, and calorie restriction. JAMA. 2007;297(9):986-94

45. Fontana $L$. The scientific basis of caloric restriction leading to longer life. Curr Opin Gastroenterol. 2009;25(2):144-50. 